CVIA

EDITORIAL

pISSN 2508-707X / eISSN 2508-7088 https://doi.org/10.22468/cvia.2018.00157 CVIA 2018;2(3):109-109

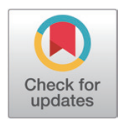

\section{A Letter for the Cardiovascular Imaging Asia Chinese Special Issue}

\author{
Bin Lu, MD \\ Chair of the Department of Radiologic Imaging, Fuwai Hospital, Chinese Academy \\ of Medical Sciences, National Center for Cardiovascular Diseases of China, Beijing, China
}

Received: June 21, 2018

Accepted: July 2, 2018
I am grateful to the Cardiovascular Imaging Asia (CVIA) editorial office for planning the Chinese Special Issue. On behalf of the five Chinese experts in cardiac imaging, we are expressing our appreciation to CVIA for providing such a good opportunity for Chinese researchers. I also thank our Chinese colleagues for the contribution of their manuscripts to this issue.

With increased life expectancy and rapid transitions in modern life style occurring across China, an epidemic of cardiovascular diseases (CVD) is emerging as a result of lifestyle changes, urbanization, and the rapidly growing aged population. Recently, CVD has become the leading cause of death in China. Cardiovascular mortality was 2.96\%o according to the Annual Report on Cardiovascular Disease in China.

Cardiac imaging as an important tool for evaluation of cardiac anatomy and heart function plays a major role in early detection of CVD. Up to the end of 2016, coronary computed tomography angiography (CCTA) was carried out in $64.2 \%$ of hospitals in China, while MRI was used in $12.6 \%$, SPECT in $11.3 \%$, and PET in $7.3 \%$. This is different from Western countries; for example, CCTA was widely used in China and become a gateway for cardiac imaging of coronary artery disease, but in western countries, more functional cardiac imaging modalities were used. To our knowledge, a total of 5 million CCTA cases a year were performed in mainland China, deriving a 20-25\% increase annually. Furthermore, various cardiac CT applications such as CT perfusion, CT-derived fractional flow reserve, and $4 \mathrm{D}$ functional analysis by cardiac magnetic resonance are now available in clinical practice. The most impressive area is Artificial Intelligence developed specifically for cardiac imaging, including automatic coronary vessel segmentation and coronary plaque and luminal stenosis analysis.

The development of cardiac imaging in China has been enhanced by many academic societies, such as Chinese Society of Radiology and Chinese Society of Cardiology, as well as many conferences and exchange and training programs. The Great Wall International Cardiovascular Congress, China Heart Congress, and Chinese Society of Radiology Annual Meeting are some of the largest and most educational cardiovascular conferences in China. Some international academic societies have been introduced into China, and branches of sub-societies have been established, such as Asian Society of Cardiovascular Imaging (ASCI), Society of Cardiovascular Computed Tomography (SCCT), and Society of Cardiovascular Magnetic Resonance (SCMR). These groups are providing an innovative and comprehensive overview of the latest research and technologic developments in cardiac imaging.

I hope this China-focused special issue will serve to emphasize the continuing enhancement and expansion of cardiac imaging in Asian counties, especially to encourage our Chinese experts and colleagues to contribute more to this growing field. 